Rev Biomed 2004; 15:1-2.

\title{
La Revista Biomédica: quince años de divulgación científica.
}

Renán A. Góngora-Biachi.

Editor, Revista Biomédica, Universidad Autónoma de Yucatán, Mérida, Yucatán, México.

Con la edición del presente número, la Revista Biomédica, publicación científica del Centro de Investigaciones Regionales “Dr. Hideyo Noguchi” y de la Facultad de Medicina de la Universidad Autónoma de Yucatán, inicia el $15^{\circ}$ año de publicación puntual y continua. Durante los catorce años como publicación científica, ha proporcionado y conservado un registro permanente de las contribuciones que se han publicado y con ello ha contribuido a la difusión del quehacer científico de la Universidad Autónoma de Yucatán, de los investigadores mexicanos y -no en pocos casos- de los profesionales de la actividad científica de Latino América y otros países del orbe. Así mismo, la Revista Biomédica ha sido un medio eficiente de comunicación entre investigadores latinoamericanos y de otros países y ha favorecido a fortalecer la identidad de grupos profesionales dedicados a la investigación científica.

Desde el inicio de su publicación en 1990 a diciembre de 2003, la Revista Biomédica ha publicado en total 1099 colaboraciones, incluyendo artículos arbitrados (revisión por pares), simposios y resúmenes de congresos (cuadro 1). El 70.6\% de las colaboraciones arbitradas han sido de origen local (estado de Yucatán), el 15.6\% de origen nacional y el
13.8\% de países extranjeros (cuadro 1). En la actualidad la Revista Biomédica tiene registrado 978 suscriptores (335 institucionales y 643 personales) cuya distribución se observa en cuadro 1.

Con el inicio del volumen 14, la política editorial de la revista sufrió algunos cambios. El principal fue que durante ese año solamente se aceptaron trabajos relacionados a la medicina humana, para con ese enfoque se transformó a la Revista Biomédica de una revista de temática biomédica general a una revista de temática de medicina humana en general. Sólo fueron aceptados para revisión por pares los artículos de medicina veterinaria que cubran tópicos relacionados con la medicina humana. Los dos últimos artículos de medicina veterinaria que estuvieron en proceso de evaluación en el año 2003, son publicados en este número.

Estos cambios fueron necesarios, ya que en el contexto de la publicación científica, por razones de registro en los índices bibliográficos, así como en la selección de las revistas por parte de los lectores, es recomendable la separación de las publicaciones de medicina humana y de medicina veterinaria. Por otro lado, esta sugerencia ya había sido emitida en una encuesta evaluadora de la Revista Biomédica 


\section{RA Góngora-Biachi.}

realizada en 2000 (1) y fue reafirmada por los miembros del Comité Editorial de la revista, así como por las autoridades académicas de la Universidad Autónoma de Yucatán.

\section{Cuadro 1}

Características bibliométricas de la Revista Biomédica (1990-2002)

\section{CONCEPTO}

RESULTADOS

\section{Colaboraciones}

Arbitradas

433

Simposios

Resúmenes de congresos

609

Origen de los artículos arbitrados $(\mathrm{n}=433)$

Yucatán México

$299(69.0 \%)$

Nacionales (México)

$72(16.6 \%)$

Extranjeros:

$62(14.3 \%)$

Distribución ( $\mathrm{n}=978$ suscripciones):

Locales

$341(35.8 \%)$

Nacionales

$553(56.5 \%)$

Extranjeros

Para nuestros colaboradores, a partir del año 2003, abrimos la posibilidad de enviar sus trabajos a través de la Internet.

Con el inicio del Volumen 15, la Revista Biomédica reafirma su compromiso de continuar con una evolución positiva (2-4), que siempre se ha traducido en mantener la calidad de su contenido, que la definan como una revista científica competitiva en el mundo de la comunicación científica.

\section{REFERENCIAS.}

1.- Álvarez-Cervera FJ, Góngora-Alfaro JL, Bata-García JL, Heredia-López FJ. Análisis de la Revista Biomédica en sus primeros diez años. Rev Biomed 2000; 11:235-49.

2.- Góngora-Biachi RA. Análisis de la Revista Biomédica. Rev Biomed 1995; 6:1-4.

3.- Góngora-Biachi RA. La Revista Biomédica: una década de su publicación. Rev Biomed 1999; 10: 197-8.

4.- Góngora-Biachi RA. Modificaciones a la política editorial de la Revista Biomédica. Rev Biomed 2003; 14:1-2. 